\title{
SPERONE SPERONI Y LA DEFENSA \\ HUMANISTA DE LA LACTANCIA MATERNA
}

\section{SPERONE SPERONI AND THE HUMANISTIC DEFENSE OF MATERNAL BREASTFEEDING}

\section{Milagro Martín-Clavijo}

Universidad de Salamanca, España

mclavijo@usal.es

https://orcid.org/0000-0001-7300-9493

Recibido: septiembre de 2021

Aceptado: noviembre de 2021

\begin{abstract}
Palabras clave: Sperone Speroni, Discorso del lattare i figliuoli dalle madri, lactancia materna, familia, maternidad, Humanismo.

Keywords: Sperone Speroni, Discorso del lattare i figliuoli dalle madri, maternal breastfeeding, family, motherhood, Humanism.
\end{abstract}

Resumen: En el Discorso del lattare i figliuoli dalle madri (1542) el humanista italiano Sperone Speroni aborda un tema de interés en el Renacimiento: si las mujeres deben amamantar a sus hijos o dejarlos en manos de una ama de cría. Se parte del análisis de este texto y se confronta con los de otros humanistas del siglo XIV -Francesco de Barbaro y Leon Battista Alberti- y del XV -fundamentalmente Erasmo de Rotterdam, pero también Juan Luis Vives, Fray Luis de León, Pedro de Luján y Antonio de Guevara- para poder entender que la defensa de la lactancia materna es uno de los elementos clave del nuevo concepto de familia y de madre en la sociedad moderna.

Abstract: In the Discorso del lattare i figliuoli dalle madri (1542) the Italian humanist Sperone Speroni approaches a topic of interest in the Renaissance: whether women should breastfeed their own children or leave them in the care of a nurse. The analysis of this text is confronted with those of other humanists of the 14th century -Francesco de Barbaro and Leon Battista Alberti- and of the 15th century -mainly Erasmus of Rotterdam, but also Juan Luis Vives, Fray Luis de León, Pedro de Luján and Antonio de Guevara- in order to understand how the defense of breastfeeding is one of the key elements of the new concept of family and mother in modern society.

\section{l. Introducción}

Sperone Speroni ${ }^{1}$ (1500-1588) es uno de los autores relevantes del Renacimiento italiano y algunos de sus diálogos -como Dialogo d'amore, Dialogo delle lingue, Dialogo

1. Sobre la vida y la producción de este autor, véase los volúmenes de Fano (1909), Camarosano (1920), Zambetti (1920). Publicaciones de años más recientes son los artículos centrados en aspec- 
della Retorica, Apologia del dialoghi- y sus obras de teatro, como Canace, han gozado de la atención de la crítica a lo largo del tiempo. Sin embargo, hasta el momento están muy poco estudiadas y, fundamentalmente no están puestas en relación con las de otros intelectuales contemporáneos, las obras en las que el humanista véneto se muestra un serio defensor de la mujer y presenta un vivo interés por distintos aspectos de la vida femenina y por el papel de esta en la sociedad italiana del siglo $\mathrm{XVI}^{2}$ : Della dignità delle donne, In lode delle donne, Dialogo del tempo del partorire delle donne, Dialogo della cura della famiglia, Dialogo d'amore, Discorso del lattare i figliuoli dalle madri y el pequeño tratado Del rimaritarsi. En estos textos, entre otras cuestiones como la defensa de la no inferioridad de la mujer frente al hombre, Speroni aborda el tema de la mujer casada, pero presentándola de una manera más positiva de lo habitual en el Renacimiento, ya que se le concede un mayor peso y dignidad dentro del ámbito familiar, aunque siempre en los límites restringidos de la esfera doméstica. La función de la madre en la sociedad renacentista la trata en varias obras, pero de manera especial en el Dialogo della cura della famiglia y en Della dignità delle donne.

En el Discorso del lattare i figliuli dalle madri ${ }^{3}$ Sperone Speroni defiende decidi-

tos más concretos del autor: Bruni (1967: 24-71), Scrivano (1959: 38-51) y Vianello (1989:203222) que recapitula los estudios hasta finales de los años ochenta.

2. Sobre la mujer y su papel en la familia en el Renacimiento, cfr. Ariès (1962), De Maio (1988), Duby y Perrot (1992), Klapisch-Zuber (1988), King (1993) y Morant (2002).

3. Las citas del Discorso del lattare i figliuli dalle madre las tomaremos de la edición de 1740 damente la lactancia materna ${ }^{4}$ frente a la práctica social en el siglo XVI de recurrir al ama de cría sobre todo por parte de las clases sociales altas. Las ideas que expresa el humanista de Padua en estas pocas páginas que componen su discurso no son nuevas ${ }^{5}$; de hecho, lo que nos inte-

(Speroni, 1740: 477-486) en la que se encuentran publicadas todas las obras de Speroni a partir de los manuscritos originales, como reza el título, y en la que se recogen sus diálogos, pero también otros textos como discursos, oraciones, súplicas, fábulas, tratados pequeños y poesías. Se considera esta edición póstuma más correcta que las del siglo XVI que, además, había sido publicada sin el permiso del autor.

4. La lactancia materna ha sido una cuestión ampliamente analizada desde distintos puntos de vista y especialmente en las últimas décadas han proliferado los estudios sobre maternidad y lactancia en el Renacimiento. Para una visión general, cfr. Fildes (1986, 1988), Klapisch-Zuber (1985: 132-164), Kohl y Witt (1978: 189-230), Matthews Grieco (1991: 15-62), McLaren (1985: 22-53), Sperling (2013), Meneghin (2010: 179195), Miller y Yavneh (2000; 2002: 90-114), Miles (2008) y Sandri (1991: 93-104).

Sobre la lactancia materna en textos renacentistas, véase Bergmann (1996: 39-49), Correll (1990: 241-62), Fernández Valencia (2011: 177205), Hairston (2013: 187-212), Nadeau (2001: 153-174) y los exhaustivos análisis de Rivera (2010: 18-32; 2016: 93-103).

5. De hecho, Naomi J. Miller (2000: 5) señala que en el Renacimiento no son pocos los tratados sobre este tema, lo que pone de manifiesto que, efectivamente, se trataba de una cuestión controvertida sobre la que no se había llegado a una conclusión mayoritaria: "treatises on breastfeeding which proliferated in a number of early modern countries conveyed class tensions associated with the use of the breast. Although the hiring of lower class women as wet nurses for infants from upper class families had a long established history, it gradually became an increasingly controversial practice, due to perceived associations between the quality of breast milk and maternal social status". En esta discusión que se mantiene 
resa es ver cómo entre los humanistas ${ }^{6}$ crece el apoyo a la crianza del niño por parte de la madre biológica, que las ideas principales para su defensa circulan entre numerosos intelectuales de la época y el porqué de este acalorado alegato. Para poder entender estas ideas que circulan en el Humanismo partiremos del discurso de Speroni para hacer referencia a otros textos que tratan de la lactancia tanto en la Italia del siglo XIV en la que esta doctrina ya se va gestando -Francesco de Barbaro en Re uxoria7 (1416) y Leon Battista

desde la época clásica llama la atención la defensa de la lactancia materna por parte de algunos humanistas. De Maio (1988: 115) señala algunos intelectuales que rechazan la leche mercenaria (Barbaro, Vegio, Alberti, Erasmo, Vives, Jacopo Sodoleto y Tansillo) y se detiene en cómo estos intelectuales se sirven de distintos mitos para convencer a las mujeres para que amamanten a sus hijos. Entre estos mitos se encuentra los clásicos -el de Isis lactans, Venus lactans, la Cabra Amaltea que amamanta a Júpiter y la Loba a Rómulo y Remo- y los propuestos por la Iglesia (la Virgen de la Leche).

6. Además de los humanistas, los sacerdotes católicos y protestantes se inclinan a favor de la lactancia materna. Margaret King (1993: 29) presenta algunos ejemplos de hombres de la Iglesia, como San Bernardino o Fray Cherubino de Siena, que consideran la lactancia mercenaria como un pecado o una práctica corrupta y abusiva. También la medicina se inclina por la leche materna argumentando que la sangre uterina se convertía en leche tras el parto, por lo que, al igual que la madre alimentaba a su hijo en el útero, lo continuaba nutriendo fuera de su cuerpo. De estas teorías defendidas por los médicos se sirven también los humanistas: se establece una relación entre el útero y los senos, por lo que la leche materna y la sangre del útero tendrían una relación directa. De hecho, de nuevo se siguen las mismas ideas ya presentadas por científicos antiguos como Galeno (Hairston, 2013: 190).

7. Para el análisis de esta obra nos servimos de la traducción del latín al italiano que lleva a cabo M. Alberto Lollio en 1548 con el título Prvden-
Alberti ${ }^{8}$ en Libri della familia (1433-34)como en otros países europeos y durante el siglo XV -principalmente interesante es

tissimi et gravi docvmenti circa la elettion della moglie. En esta obra Barbaro trata el tema general "del modo che si debba tenere, in alleuare e costumar i figliuoli (...) parte non solo utile, ma graue, e di gradissima importanza" (1548: 58v). En el capítulo 18 de esta edición el autor defiende la lactancia materna de forma decidida ("Prego le donne tutte, a uoler per ogni modo co'l latte lor medesimo alleuare i propri figli" (1548: 60r) y se aleja de los escritores medievales como Aldobrandino da Siena, Francesco da Barberino y Paolo da Certaldo que, en general, siguen los textos antiguos, defienden la lactancia materna y animan a las madres, pero, a su vez, reflejan de forma clara la costumbre social de servirse de un ama de cría, excusan a las madres que no lo hacen y, al final, dedican más espacio a cómo elegir a la mujer que va a criar a sus hijos.

Sobre la lactancia en la época medieval, véase Hairston (2013: 196-200). En relación con la lactancia materna, Barbaro funciona como un puente entre los textos precedentes y el de Alberti.

Sobre Barbaro y las obligaciones de la mujer, cfr. Kohl-Witt (1978: 179-230).

8. Las citas que aparecen en este artículo del libro primero de I libri della famiglia se han tomado de Alberti (1960: 13-82). Este volumen recoge conversaciones entre varios miembros de la familia Alberti en Padua en 1421. El autor ya no se sirve del latín como Barbaro, sino que lo hace en italiano y se dirige a un público más amplio. En el primer libro se ocupa en pocas páginas de quién tiene que criar al niño y es el personaje de Lionardo, joven soltero, el que defiende la crianza del niño a cargo exclusivamente de la madre, por tanto, es él el portavoz de la posición teórica de los humanistas sobre la lactancia materna en contra del parecer de su hermano casado y con hijos, Adovardo, que presenta la práctica social.

Sobre I libri della famiglia, cfr. la introducción de Guido A. Guarino (1971: 7-26). Sobre la lactancia en Alberti, y más en concreto sobre las contradicciones y tensiones que se pueden observar en el discurso de Alberti sobre la lactancia, véase Hairston (2013: 203-211). 
Erasmo de Rotterdam ${ }^{9}$ y el coloquio Puerperio (1526), pero también algunos autores españoles como Juan Luis Vives en La instrucción de la mujer cristiana (1523), Antonio de Guevara en Relox de príncipes (1529), Pedro de Luján, Coloquios matrimoniales (1550) y Fray Luis de León con La perfecta casada (1583).

Con respecto a los modelos antes del Humanismo, desde las primeras páginas del discurso Speroni señala explícitamente de dónde ha tomado esas ideas: ha escuchado "una orazione volgare" (Speroni, 1740: 477) al filósofo averroísta Marcoantonio de' Passeri (Padua 1492-1563), Ilamado Gènua, Genoa o Genova, en la academia degli Elevati de Padua. A su vez Genova, como lo cita Speroni, había tomado los argumentos de un "buono antico Romano" (477) y también de Favorino de Arlés, filósofo griego sofista. En estas primeras páginas Speroni insiste mucho en que no se trata de sus propias palabras (quizás, como veremos, por el tono contundente de crítica a la práctica social), que él solo las repite y que lo hace con buena intención, con un noble fin: se trata de un dis-

9. El coloquio Puerperio de Erasmo de Rotterdam se publicó en Familiarum colloquiorum formulae en la edición de 1526. Aquí nos servimos de una edición actual a partir de la traducción que en 1529 Alonso Ruiz de Virués realizó de este diálogo como parte de Coloquios familiares, probablemente la obra de Erasmo más leída en la España del siglo XVI. En este texto también se defiende la obligación de la madre de alimentar y de criar a su hijo. Para Rivera (2010: 29) "la conducta paradigmática de la madre formulada en Puerperio ocupa un puesto significativo en las prácticas discursivas del género y la familia y en la construcción de las nociones de la domesticidad femenina". Los protagonistas son Jocundo y Sofía: ella es una joven madre que ha decidido contratar a una ama de cría para su recién nacido; Jocundo es un amigo de Sofía que intentará convencerla para que amamante ella misma a su hijo. curso dirigido a una joven madre a la que el autor considera como su hija y a la que aconseja como tal: "se lette furono pubblicamente, ed ascoltate da assai persone, ed io fra tutte me l'ho recate nella memoria (...) Mio è bene il buono animo" (477-8).

\section{Naturaleza vs. práctica social}

Speroni considera que, ya que, con respecto al hijo, la madre lo ha hecho todo casi sola (engendrar, gestar y parir), no va a necesitar asistencia alguna tampoco a la hora de alimentarlo: "Non fu quella essa senza altra aggiunta, che cominciò esser madre, ed a ciò fare su posto in opra suo corpo solo? .... perché la cerca [la ayuda] al nutrire?" (1740: 484). Esa ayuda externa iría contra natura, mientras que es la naturaleza la que le tiene que servir como guía, ya que ha provisto a las mujeres de todo lo necesario para criar a los hijos; por tanto, continúa Speroni, "risponda dunque nel proprio corpo al principio il fine e il mezzo dela sua impresa, nè torca i passi della natura la mala usanza del vulgo reo" (484).

En Puerperio Erasmo también considera que criar a los hijos es obligación de la mujer y propia de su constitución y no hacerlo es contradecir abiertamente la naturaleza; a la mujer se la compara con la tierra que es madre por encima de todo y que no solo engendra, sino también se ocupa de sus criaturas después de nacer: "¿Por qué la tierra es llamada madre de todas las cosas?, no por cierto solamente por engendrallas, sino porque, después de engendradas, las ceva e cría con la mesma fertilidad e virtud que las engendró" (2005: 26).

En Libri di famiglia Alberti (1960: 31-32) también se manifiesta en el mismo sen- 
tido a través de las palabras de Lionardo ("Sempre veggo la natura da ogni parte sollecita a provedere che ogni cosa procreata sé stessi conservi, ricevendo da chi la produsse nutrimento e aiuto a perseverare in vita e a porgere le sue utilitati in luce") y presenta muchos ejemplos en que la naturaleza se comporta como verdadera madre: "Veggo nelle piante e arbuscelli quanto le radici attraggono e distribuiscono alimento al tronco, el tronco a' rami, e' rami alle frondi e a' frutti. Così forse sarà da stimare naturale a' padri che nulla lascino adrieto per nutrire e mantenere quelli che sono di sé usciti e per sé nati" (32).

Uno de los puntos fuertes de la defensa de la lactancia materna radica en que ha sido la propia naturaleza la que ha hecho que las mujeres tengan pecho, al igual que ocurre con los otros mamíferos. Esa parte del cuerpo no se ha creado de adorno: la naturaleza es sabia y provee siempre de lo que es necesario; Erasmo también lo recalca: "Pues vees en tu pecho esas dos fontezillas de leche que parecen querer rebentar, entiende que naturaleza no te las dio en vano, sino para avisarte del oficio que te quedas por hazer después que pariste" (2005: 42).

De la misma manera Alberti aboga por seguir a la madre naturaleza en todo momento, aunque sus directrices, a veces, sean opuestas a las de los médicos:

Forse questi medici appongano che dare el latte le indebolisce e falle talora sterile. Ma pure io posso credere dalla natura sia bene a tutto proveduto, e debbasi stimare non sanza cagione, ma bene con gran ragione quanto si vede insieme colla grossezza ivi nascere in copia e multiplicarsi el latte, quasi come la natura stessa ci apparecchi al bisogno e dicaci quanto a' figliuoli dalle madri aspetti (1960: 36-37).
Por su parte, Francesco Barbaro insiste en De re uxoria que si la naturaleza ha dado mamas a las mujeres es fundamentalmente para poder nutrir a los hijos: "ad esse specialmente ha dato l'ufficio e del partorire, e dello alleuare i figliuoli" (1548: 59r). Además, añade que la naturaleza se ha comportado todavía mejor con ellas, porque, al contrario que otras hembras, las mujeres tienen las mamas en el pecho y no en el vientre y es así para que puedan abrazar y besar a sus hijos al mismo tiempo que le dan la leche (59r). De esta manera, la madre se convierte en el único ser capaz de satisfacer las dos necesidades más importantes del niño (y del ser humano): alimento y amor. Si la naturaleza lo ha querido así, la mujer debería seguir a la naturaleza y amamantar a sus hijos.

Estos mismos argumentos los presentan también Vives en Instrucción de la mujer cristiana $^{10}$, Antonio de Guevara en Relox de príncipes ${ }^{11}$ o fray Luis de León en $L a$ perfecta casada ${ }^{12}$.

Si los intelectuales humanistas dedican tanto espacio a la defensa de la lactancia materna es porque realmente lo habitual entre las clases más altas (pero ya en el siglo XV también en las familias de cla-

10. “¿Piensas por ventura que naturaleza dio de balde tetas a las mujeres y que puso allí aquellos dos pezoncicos como dos berrugitas no por más de por una cierta gentileza o hermosura de los pechos? (...) No lo hizo sino a fin que la madre habiendo parido tuviese con qué poder criar a su hijo, según hacen todas las otras alimañas" (Vives, 1940: 138).

11. "Porque naturaleza no sólo hizo ábiles a las mugeres para parir, pero juntamente con esto las proveyó de leche para criar" (Guevara, 1994: 507).

12. La naturaleza "declara casi a voces su voluntad, enviando luego después del parto leche a los pechos" (Fray Luis de León, 2006: 139). 
se media con un alto número de mujeres que entran en el mercado laboral) era lo que se denominaba "latte mercenario o baliatico" 13 .

Sobre esta práctica social extendida en Europa Margaret King (1993: 30) afirma, aportando mucha documentación, que "los hijos de las mujeres ricas eran alimentados por los pechos de las pobres: así como las primeras estaban condenadas al "embarazo perpetuo», las últimas estaban condenadas a la «lactancia perpetua»". De hecho, esta costumbre dividía a las mujeres en dos grupos: las que se dedicaban fundamentalmente a parir y las que se ocupaban de la crianza de los niños. Además, era muy habitual que no fuera el ama de cría la que se estableciera en la casa del recién nacido, sino que se enviara al niño al campo durante el tiempo de la lactancia; una distancia que no favorecía que los padres biológicos lo visitaran a menudo ${ }^{14}$. Teniendo en cuenta también la alta mortalidad infantil, muchos de estos niños no volvían nunca a su casa, y los que lo hacían no habían disfrutado del amor de sus padres durante los primeros años de su vida. Además, muchas veces ni siquiera estaban siempre con la misma ama de cría. Un último e importante aspecto sobre esta práctica social lo recalca King (1993: 33) con rotundidad: "Las nodrizas en la sociedad europea (con excepción de las esclavas) sólo lactaban por dinero". En este aspecto económico, que se encuentra en la base de la relación entre ama de cría y niño, insistirán también los intelectuales humanistas a favor de la lactancia materna.

13. De hecho, Hairston (2013: 189) constata una evidente contradicción entre la literatura prescriptiva humanista y la práctica social.

14. Sobre la condición de los niños con ama de cría, cfr. De Maio (1988: 116).
Speroni se refiere a este uso social bien arraigado en la sociedad renacentista como "fera usanza maligna (...), usanza nel vulgo nata, non altramente che nascer sogliono di vil materia corrotta zanzare e vermini fastidiosi, ogni onorata matrona senza altro intender volerne, dovrebbe avere in dispetto" (1740: 479). Erasmo también se muestra contrario a esta costumbre que no es racional, sino más bien una moda que carece de toda base natural. Sofía, la puérpera protagonista del coloquio de Erasmo, se atrinchera en esa práctica que se ha convertido casi en una obligación y que se sigue sin apenas reflexionar sobre ello: "Úsase así ya en todo el mundo" (Erasmo, 2005: 25). A estas palabras, Jocundo, con rotundidad, responde: "Parécete que sería suficiente escusa para hazer qualquiera cosa destas decir: todo el mundo lo haze?" (26).

Entonces, ¿por qué en general las mujeres de la élite europea decidían no amamantar a sus hijos ${ }^{15}$, a pesar de que durante siglos numerosos médicos, sacerdotes e intelectuales lo desaconsejan decididamente y se sirven de autoridades clásicas para fundamentarlo? Podemos entender que no se trataba de un simple capricho o únicamente del deseo de librarse de una pesada carga que recaía únicamente sobre ellas durante años. Entendemos que esas madres querían a sus hijos y que no decidían sin más apartarlos de ellas: tenía que haber importantes motivos que llevaban a las madres y, por supuesto, a los padres, ya que eran también ellos los que en última instancia tomaban esa decisión, a no amamantar a sus hijos.

15. King (1993: 28) presenta un ejemplo concreto extraído de textos sobre las vidas de santos: de una amplia muestra de madres de santos, provenientes, en su mayoría, del estrato social más alto, solo unas pocas amamantaron a sus hijos. 
Una primera razón para delegar la crianza en otra mujer la presenta Sofia: ella es muy joven, por lo que no es indicado dar el pecho: "Esto a parecido a mis vecinas e amigas, porque no me pusiesse en tan tierna edad al trabajo del criar" (Erasmo, 2005: 26). Jocundo no considera justificable renunciar a esa función materna simplemente para evitar a la mujer un esfuerzo, una fatiga que podría repercutir en su salud y, de nuevo, apela a la naturaleza: "La edad que dio fuerças para engendrar, darla ya para criar lo que engendraste" (26).

Por otro lado, en el Renacimiento se consideraba que mientras daban el pecho las mujeres no podían mantener relaciones sexuales, ya que esto incidía negativamente sobre la calidad de la leche y podía poner en serio peligro la salud del bebé16 . De esta manera, el comportamiento sexual de la madre afectaba directamente tanto a su propia integridad como a la del niño. Por esto, la mujer que daba el pecho no podía mantener relaciones con el marido durante los meses (o los años) en los que continuara con la lactancia. Morant (2002: 186) añade que no pocos sacerdotes defendían que las madres no amamantaran a sus hijos para que estas pudieran mejor atender las demandas sexuales del marido ${ }^{17}$. Además, durante

16. Alessia Meneghin ha estudiado esta cuestión centrándose fundamentalmente en la sociedad florentina y también ha concluido, citando a Klapisch-Zuber (1985: 132-64), que "sexual activity and a new pregnancy would spoil the milk for the nursling, who might die if he or she fed from the pregnant mother". (2010: 180).

17. De hecho, en el Renacimiento impera la doctrina del débito conyugal, es decir, la obligación que tenían los cónyuges de satisfacer los deseos carnales del otro. Sobre esta doctrina y su aplicación en el Renacimiento, véase Morant (2002: 257). la lactancia muchos esposos se lamentaban de que sus mujeres estaban menos limpias, que olían y, en general, se descuidaban más, por lo que muy a menudo preferían satisfacer sus necesidades fuera del hogar (Morant, 2002: 186). De esta manera, la lactancia materna ponía en serio peligro la unidad familiar.

Otra razón importante a favor de recurrir a la ama de cría tenía que ver con la fertilidad $^{18}$ : las mujeres, sobre todo de las clases sociales altas, debían asegurarse muchos hijos, herederos para la familia, $y$, dada la elevada mortalidad infantil de la época, una lactancia prolongada retrasaba la posibilidad de un nuevo embarazo. Si tenemos en cuenta, tal y como se afirma en numerosas obras sobre el papel de la mujer en el Renacimiento, entre ellas los textos de Leon Battista Alberti y Francesco Barbaro, que una de las cualidades por la que se elegía una esposa en el Renacimiento era precisamente que fuera

18. Sobre la obligación a la fertilidad en la mujer renacentista y una vida marcada por los ciclos de parto y lactancia, véase King (1993: 15). La estudiosa británica declara que solo los periodos de lactancia libraban a la mujer del peso continuo de los embarazos y partos, que las mujeres pobres daban a luz cada 24-30 meses, pero que eran las ricas las que tenían más hijos precisamente porque no solían amamantarlos. Por tanto, eran las mujeres de clases altas las que tenían una mayor presión para la procreación y esa era su función primordial. También Sandri es de la opinión de que la lactancia era considerada un método para no quedarse embarazada y que ponía en peligro el papel de esposa y sus obligaciones como tal: "La donna-madre veniva a trovarsi divisa da una duplice incomparibile responsabilità: l'allattamento dei figli da una parte e l'adempimento del dovere coniugale dall'altra. È evidente che il timore di incorrere in inevitabili peccati contro natura inclinava i genitori -consenziente la Chiesa- alla scelta dell'allontanamento dei figli mediante il loro affidamento a balie mercenarie" (1991: 101). 
fértil, podemos imaginar que recurrir a un ama de cría para la lactancia de sus hijos no era, ni mucho menos un capricho para estas mujeres.

Todos estos motivos llevaban a las mujeres a delegar su función alimenticia en terceras personas fuera del hogar y a que se convirtiera en algo tan habitual que llevaba a muchas madres, como señala Barbaro, a avergonzarse si amamantaban a sus hijos: "Non hanno le madri a uergnarsi (some sogliono fare a nostri tempi) di nodrire co'l proprio latte i propri figli" (1548: 59v).

\section{De medias madres, madres incompletas y monstruos inhumanos}

Como esta práctica social está tan arraigada en la sociedad, los humanistas se muestran inflexibles, y a veces instan, con duras palabras, a las madres para que vuelvan a la ley natural y den el pecho a sus hijos. Hablan así de corrupción de la nobleza del cuerpo y de la mente del neonato, de medias madres, de madres incompletas, de madres monstruosas e incluso de monstruos inhumanos. Si tenemos en cuenta que la mujer era tal en cuanto esposa y sobre todo como madre, el no cumplir con una de sus obligaciones primarias, la de amamantar a sus hijos, las rebaja a ser menos que mujer, a no ser nada.

Speroni no se aparta de este filón humanista en defensa de la lactancia materna y se muestra también muy contundente a la hora de calificar a estas madres que, por decisión, no por necesidad, recurren a un ama de cría: "Qual di noi per elezione (perchè a destino non ha contrasto la nostra debole umanità) dell'altrui latte fa nutricare i figliuoli, tale in questo atto non ama quelli nè se; e come mostro inumano privo del senso e dell'intelletto, non è degna di dirsi madre, nè di esser viva" (1740: 480). Como vemos, el intelectual de Padua califica este acto como de falta absoluta de amor por parte de la madre que la convierte en un monstruo que ha perdido cualquier connotación humana; por eso, no solo no son dignas de llamarse madres, sino que incluso considera que no deberían ni siquiera seguir vivas. Es quizá esta radicalidad, este uso de términos muy duros y elegidos conscientemente, el que le ha llevado a recalcar al inicio de su discurso que esas palabras no son suyas, aunque las comparta totalmente.

Speroni disponía de una amplia gama de argumentos desde la época clásica ${ }^{19}$ de la que servirse para intentar concienciar, y convencer, a las mujeres y son esos autores los que ya utilizan expresiones como "media madre" o "madre incompleta" para designar, con gran desprecio, a las mujeres que no dan el pecho a sus hijos.

Como harán también otros intelectuales, como Alberti, para la defensa de la lactancia materna es necesario recurrir a los antiguos $^{20}$ y de obligada citación es el escritor latino Aulio Gelio, quien en Noches Áticas a su vez se sirve de un discurso de su maestro Favorino de Arles. La situación es muy parecida a la que presenta Spe-

19. Sobre la lactancia en la Antigüedad, tanto a favor o en contra de las madres que amamantan a sus hijos, véase Hairston (2013: 193-195) que analiza este aspecto en autores como Plutarco De liberis educandis-, Soranus de Efeso-Gynecology-y Galeno-De Sanitate tuenda.

20. En la primera centuria después de Cristo nos encontramos ya con el decreto de Marco Aurelio en el que se afirma que la mujer es madre completa solo cuando da a luz y alimenta personalmente a sus hijos. 
roni: el filósofo griego había ido a visitar a la mujer de un discípulo que acababa de tener un hijo y que había decidido no amamantarlo. Favorino considera que esta práctica va contra natura y que si una madre priva a su hijo de su propia leche se la considerará "mater dimidiata"21: una mujer que no se realiza completamente como madre y que, por tanto, tampoco lo hace como mujer de forma plena; solo criar personalmente a los hijos convierte a la madre verdaderamente en una madre (los nueve meses en el vientre y el parto es solo una parte de la esencia de la maternidad); de no ser así, la mujer está condenada a ser una madre imperfecta, una mujer no completa. Para Gelio la lactancia materna es lo natural para una mujer e "identifica nella funzione nutritiva mediante l'allattamento ciò che definisce l'identità femminile" (Basile, 2016: 175). Esta equivalencia entre identidad femenina y crianza del hijo es fundamental para entender la presión a la que se sometía a estas mujeres.

Para que ese hijo sea virtuoso es fundamental que lo críe la madre, de hecho, es su responsabilidad y obligación exclusiva no solo llevarlo en el vientre y parirlo, sino también amamantarlo y criarlo. El modelo que estas madres tienen que seguir es siempre el de la naturaleza que engendra, pero también que nutre siempre a sus criaturas: "Tu accrescendolo e nutricandolo gli dei lavar col tuo latte, e fornirai il tuo dovere (...) comunemente crede ogni femmina, che 'I portar solo de' figlioli sia naturale oprazione, e voler libero il nutricarli (...) le quai cose mal si convengono

21. Basile (2016: 171-176) estudia desde el punto de vista filológico las palabras con las que Gelio define a esta madre para poder comprender hasta el fondo la implicación que tenía ser considerada media madre en la sociedad de la época. colla intenzione della natura" (Speroni, 1740: 482-483). La maternidad continúa mientras el niño necesite a la madre, por eso, quien no lo alimenta es una madre a medias: "Ora che hai partorito, se non fai altro per la salute di tuo figliolo, sei mozza madre e non più: nè sei per esserli madre intera, se non allora che interamente, quanto a te tocca, l'arai nutrito" (480). De hecho, uno de los aspectos cruciales de esta defensa de la leche materna se encuentra en la definición de madre: no se trata solo de la que engendra, lleva en el vientre durante nueve meses y después da a luz, sino también la que lo cría una vez fuera de su cuerpo: "Tu che tale l'hai partorito, tale il dei conservare" (482). Con estos argumentos, los humanistas dan una gran importancia a la maternidad después del parto, amplían las funciones de la madre y le dan mayor dignidad. De la misma opinión es Erasmo: "No pienses que has acabado de parir hasta que tu hijo ayas confirmado en las fuerças del cuerpo e fortificado en las virtudes del alma" (Erasmo, 2005: 45). Así también lo cree Speroni:

II fanciullo in tale stato si trova essere, che non può nulla da $\mathrm{se}^{22}$, ed ha bisogno di nutrimento, che gli si porta dalla nutrice o da te (...) Vuol natura, vuol ragione, vuole onestà, che 'I nutricarlo sia pur da te, che "I generasti, che 'I portasti, che 'I partoristi: ma fera usanza maligna porta in contrario (Speroni, 1740: 479).

Naturaleza, razón y honestidad confluyen en esta necesidad de alimentar personalmente al hijo. Es más, se considera que es todavía más importante conservar al hijo en vida a través de la nutrición y el cariño que traerlo al mundo. Así lo expre-

22. Es por ello que Speroni (1740: 481) habla del niño como de "fanciullesca imperfetta" y que es la madre la que le debe llevar a la perfección. 
sa Speroni: "Vuol natura (...) che assai più vaglia la cosa fatta, che non valeva in facendosi (...) però stima più il fanciullo poiché egli è nato, che non faceva quando era a nascere, e più il salvarlo che 'I generarlo; per conseguente più il nutrire, che 'I partorire non facea" (1740: 481).

Erasmo de Rotterdam también cuestiona que se pueda llamar madre comple$\operatorname{ta}^{23}$ a alguien que delega una función tan importante como la nutrición en una extraña ${ }^{24}$, una mujer que no pertenece

23. Rivera (2016: 21) en su estudio sobre el coloquio de Erasmo Puerperio subraya que el término madre incompleta o media madre "Jocundo lo emplea para destacar la fragmentación que sufre la identidad de la madre, y en consecuencia la del hijo, como resultado de la división del parto y del amamantamiento en dos mujeres diferentes". Palabras muy parecidas utiliza Guevara: "ca la muger es media madre por el parir y es media madre por el criar" 1994: 511). Sobre la figura de la madre monstruosa en Guevara, véase, Bergmann (1996: 39-49).

24. Erasmo aporta numerosos ejemplos de la madre naturaleza que no deja a sus hijos en manos de una extraña, que no trasplanta a sus hijos, no le echa de sí: es el caso del "trigo que, echado en mala tierra, pierde su nativa bondad o el de las plantas que, arrancadas de la tierra, se mueren" (2005: 27). Para el humanista holandés dejar que a tu hijo lo alimente una ama es como comprar una cabra para criar a tu hijo (43). De hecho, nadie podrá amar a un hijo ni criarle tan bien como una madre. Vives también habla de leche extraña: “¿Qué desdicha es esta tan grande y estragar la nobleza y valer del hombre recién nacido y al ánimo y cuerpo suyo formado por tan hermosos principios, corromperle con embastardido y enjerido mantenimiento de leche extraña?" (1940: 139). Luján en Coloquios Matrimoniales incide también en que no se debe dejar al hijo en manos de extrañas: "parece cosa muy monstruosa que haya ella parido a la criatura de sus entrañas y que otra mujer extraña le de sus tetas" (2010: 133). Sobre la condición de gente extraña reincide también Guevara: "porque mamando el niño a la familia, con la que no hay lazos de sangre. En Centuries of Childhood: A Social History of Family Life Ariès explica Ios cambios en la concepción de la familia durante este periodo y señala que "The family ceased to be simply an institution for the transmission of a name and estate -it assumed a moral and spiritual function, it moulded bodies and souls" (Ariès, 1962: 412). En esta nueva concepción de la familia como institución principal de la sociedad que hay que proteger a ultranza entra de lleno la defensa de la lactancia: hay que intentar por todos los medios que los niños no se críen con extraños, que de su educación y alimentación se ocupe la madre fundamentalmente y se haga en el hogar familiar y no fuera de él. Hay que asegurarse así el rígido control del núcleo familiar. En este sentido reportamos el diálogo entre Jocundo y Sofía:

Joc. Dime, ¿sientes por muy dulce el nombre de madre?

Sof. Sí siento.

Joc. ¿Consentirías, si hazerse pudiese, que otra muger fuesse madre de lo que tú pariste?

\section{Sof. En ninguna manera.}

Joc. Pues ¿por qué te determinas a transferir en otra muger estraña más de la mitad del nombre de madre?

Sof. Nunca Dios tal quiera; yo no reparto mi hijo, ante todo quiero ser enteramente su madre. (Erasmo, 2005: 26).

Como vemos las palabras de Jocundo ponen a la joven madre contra la pared acusándola de compartir con una extraña su condición de madre; “¿Qué harías si ya, quando sepa hablar, en lugar de madre, te llamasse media madre? ¿No le açotarías? Pues menos eres que media madre a la

leche de mujer agena, impossible es que tome la condicion de madre" (1994: 510). 
hora que rehusas de criar lo que pariste" (43). Más adelante, vuelve a la cuestión de la media madre y apela al afecto materno de Sofia, pues a los niños no solo los cría la leche, sino "el abrigo e calor de las madres" (43). Si se aparta al hijo y se entrega a un ama de cría, este no recibe ni su alimento ni su calor humano, por tanto ¿cómo podrá el hijo luego considerarla madre?

"Quando començare a crecer; ni él tan fielmente te obedecerá, ni tú con tanto cuydado mirarás por él, si comienças a sentille algunos respectos e condiciones mamadas con la leche, que representen, no a tu nobleza, sino a la poquedad e servidumbre de la ama que lo hubiere criado, como en algunos lo emos visto" (2005: 44).

Por todo ello, la madre no puede apartarse de la naturaleza que es la que debe guiar todo tipo de comportamiento; si ella no se conforma con la naturaleza, será una madre a medias, una madre imperfecta; además, señala Speroni, en el caso de que la madre no dé el pecho a su hijo por capricho o por su propio beneficio, se la podría considerar una mala mujer ${ }^{25}$. Realmente solo en ese caso la madre se convierte en monstruo incivil y enemiga de la humanidad:

Queste sono le condizioni date alla donna dalla natura per farla madre: delle quali quandunque manca una sola, (e ciò è caso, o destino che dir si voglia) senza alcun dubbio questa cotale è sempre madre imperfetta, non però indegna, che le sia avuta compassione. Ma se il difetto è da lei, che poco pregi il figliolo, o ami troppo i suoi agi; chiaro mostra la mala femmina, che fatta

25. Speroni compara a esas malas mujeres con animales salvajes: "La mala femmina in cotale atto dall'esser suo tramutandosi, alla maniera di Anassarete o di Niobe, di donna sasso esser fatta, o lionessa come Atalanta, o qual Calisto di Licaone esser cangiata in una orsa" (1740: 486). è madre contra sua voglia, e che solo per suo diletto senz'altro fine si maritasse; onde quanto al suo reo animo, sia meretrice e non moglie, e come tale, cioè non donna, ma fero mostro incivile con fronte umana e cor nemico d'umanità, sia giustamente d'infami degna e di pena (Speroni, 1740: 480).

Speroni se sirve de expresiones peyorativas como "quitar la leche a sus hijos" o dejarles en manos de "personas extrañas" para presentar a estas madres casi como delincuentes que utilizan la crueldad de manera gratuita ${ }^{26}$ frente a las personas que más se deberían querer y que más lo necesitan. El humanista italiano acude a fuentes clásicas como Platón, Sócrates, Gorgias y Protágoras para llegar a las siguientes conclusiones que califica como "extrañas": "la prima è questa, che la villana diventa madre del gentiluomo nel nutricarlo; l'altra allo 'ncontro, che la madre che 'I partoritte, nel dipartirlo dal proprio latte e darle a bere l'altrui, perde il nome dell'esser madre, e si tramuta in matrigna" (Speroni, 1740: 485).

En La perfecta casada Fray Luis de León también califica de madrastras a estas madres que van contra natura y atribuye el término de madre a las amas de cría, ya que las primeras llevan a su hijo nueve meses en el vientre, mientras que las segundas los crían durante al menos dos años: "el ama es la madre, y la que le parió es peor que madrastra, pues enajena de sí a su hijo y hace borde lo que había nacido legítimo ${ }^{27}$, y es causa que sea mal nacido el que pudiera ser noble" (2006: 137).

26. En este sentido, Speroni considera la no lactancia como una barbaridad propia de pueblos salvajes, como los turcos o los moros (1740: 485). 27. Como vemos, recurrir a un ama de cría podría perjudicar seriamente la legitimidad del hijo, convirtiendo un hijo legítimo en uno fuera de la 
Por todas estas razones, para Speroni estas madres a medias deberían incluso ser castigadas:

Sarebbe adunque in un buon rettore miglior giustizia, se egli punisse la rea femmina che niega il latte al figliolo, e giunto il tempo di nutricar lo innocente, dà lui bando del proprio petto, il quale è patria e rifugio suo, che non fa ora nel castigarla, perchè dal ventre il diparta innanzi il tempo di partorire (1740: 481).

Barbaro también comparte la misma opinión y aboga por el castigo: "Di grauissimo castigo adunque saranno degne le madri, se la cura e il gouerno de lor figliuoli non haueranno a cuore. Però elle non debbono ricusar fatica alcuna, per alleuargli, e instruirgli" (Barbaro, 1548: 59v).

Otro de los argumentos que presentan los intelectuales humanistas para defender la lactancia materna es que el niño se va a encontrar con dos madres, no con una. La consecuencia primera es evidente: la madre fisiológica se va a sentir menos querida por su hijo que preferirá a la que le cría y pasa el día entero con él. Así se lo hace ver Jocundo a Sofía en Puerperio:

Menos te amará tu hijo repartiendo el amor filial en dos madres, pues que todos los criados a sus amas llaman madres, e por tales las tienen, e tú, por consiguiente, no lo podrás tener tan entero amor como si sola oyeses de su boca el nombre de madre. Allende de esto, quando començare a crecer, ni él tan fielmente te obedecerá, ni tú con tanto cuidado mirarás por él (Erasmo, 2005: 44).

Además, la madre fisiológica tendría menos posibilidad de que su hijo siguiera sus consejos y, en general, de influir sobre su formación y su comportamiento: "Que aya una cierta confederación de amor entre el que enseña y el enseñado, entre el que

familia, de alguna forma contaminado por la bajeza de la mujer que lo nutre. aconseja y el aconsejado, por lo qual, si ninguna cosa del natural amor entre tu hijo e ti se menoscabare mejor le podrás infundir los saludables consejos" (44). Esto es muy importante porque se considera que en estos primeros años de vida la madre debe cumplir también con la función de educadora ${ }^{28}$, de manera especial en valores morales y en buenas costumbres durante los primeros años de la infancia. De hecho, los padres cumplen con la importante misión de iniciarles en los estudios solo cuando los hijos ya han crecido un poco ${ }^{29}$; en este sentido, Erasmo habla de trasplantar al hijo, de separarle de la madre tras esos primeros años para que sea el padre el que se ocupe de darle otro tipo de nutrición, la de la mente (27) ${ }^{30}$.

En esta línea de acusaciones, Speroni llega a comparar a la madre que no cría personalmente a sus hijos con una que aborta voluntariamente, es decir, expulsa, aparta al hijo recién nacido de sí y le priva del amor y del alimento materno. Erasmo establece la misma comparación:

No solamente no es entera madre la que no cría a su hijo, más aún, ni se puede dezir

28. Sobre la diferencia entre la educación materna en niños y niñas, véase Morant (2002: 87).

29. Speroni no se olvida de reivindicar la necesidad del padre a la hora de concebir un hijo ("È vero che al generare de' figlioli ambi insieme concorrono la madre e il padre ugualmente" [1740: 479]). y de llevarlo más tarde a su perfección.

30. Barbara Correll (1990: 251) señala las distintas funciones asignadas por separado al padre $y$ a la madre: "As mother the woman nourishes the body of her (male) child until she transfers 'nurturing' responsibilities to the father, who (when the child reaches age seven) looks to education and 'harder lesson, which are the father's responsibility rather than the mother's. It is the mother, grounded in the maternal body, who nurtures the infant's body as an 'instrument to the mind,' until formal and manly education takes over". 
avelle parido, sino movido, pues que como abortivo luego le echa de sí, y en tales mugeres se assienta bien la derivación de nombre de madre que suelen dar los griegos, por la qual sinifican que no guardan lo que paren. Ca, sin duda, dar el niño rezién nacido, e antes que aya perdido el calor de la madre, a una muger alquilada para que le críe, genero de desamparalle es. (43).

Por todo ello, para el humanista de Padua, esas madres que no dan el pecho deberían ser castigadas de igual modo que las que abortan: "Usa alcuna arte per sgravidare; il magistrato ciò intendendo suol castigarla agramente: e fa di ciò un gran bene" (1740: 481).

Poco a poco todos estos argumentos van a conducir a poder calificar a las madres que dejan que sus hijos los críen otras mujeres como de monstruos. En general, los humanistas establecen un vínculo muy fuerte entre alimentar y querer y consideran monstruoso no ver crecer al hijo y entregarle su amor cada día. De hecho, es durante los meses de lactancia cuando se fortalecen los lazos de afecto entre madre e hijo, tal y como afirma Alberti en el primer libro de I libri della famiglia: "Quale con più amore, con più fede, diligenza e assiduità governerà el fanciullo, o quella condutta per pregio, o la propria madre. Né ancora mi stenderò a provarti quanto l'amore verso del figliuolo si conservi e confermi alla madre quando el figliuolo sarà nel suo seno cresciuto e nutrito" (Alberti, 1960: 37-38).

\section{Contra las amas de cría}

Como hemos visto, no son pocos los humanistas en Europa que apuestan con decisión por una mayor implicación de la mujer en sus funciones maternas, pero hasta ahora hemos analizado solo las partes de sus obras en las que estos intelectuales instan a las madres a dar el pecho. En otros momentos de los mismos textos, estos intelectuales se muestran también en contra de las amas de cría y las rechazan radicalmente: consideran que acudir a ellas es una costumbre no racional, incluso bárbara, en la que la contraparte, el ama de cría, no es otra cosa que una "mujer alquilada" que se ocupa del niño exclusivamente por dinero ${ }^{31}$. Al fin y al cabo, como pregunta Jocundo a Sofía:

¿De dónde piensas que viene tu hijo, sin saber hablar, casi comiença ya a demandar la deuda que naturalmente le deves, Ilamándote mamá? ¿Cómo le puedes oyr boz mal formada entre los tiernos paladarejos, sin dalle luego a mamar; y échasle a que se lo de otra mujer alquilada, como si comprases alguna cabra para criar a tu hijo? (Erasmo, 2005: 42-43).

En muchos tratados en los que se discute el tema de la primera nutrición del niño se hace referencia fundamentalmente a cómo elegir una buena ama de cría ${ }^{32}$ y,

31. En este aspecto monetario insiste Erasmo: "E quando esto tenga, sabes que hará más cuenta de ganar un maravedí que de la vida de tu hijo" (Erasmo, 2005: 27).

32. Un caso evidente es I libri di famiglia, volumen en el que Adovardo hace una defensa del ama de cría y señala cómo hay que elegirla para evitar que se perjudique al niño: "Convienci pensare molto innanzi a ritrovare buona balia, cercarne con molta opera per averla a tempo, investigare ch'ella non sia inferma né scostumata, e porvi mente e diligenza ch'ella sia vacua, libera e netta di que' vizii e di quelle macule quali infettano e corrompono il latte e il sangue; e più abbiamo da procurarla tale che in casa seco porti né scandolo né vergogna" (Alberti, 1960: 34). Alberti señala también lo difícil que es encontrar una buena ama de cría y el tiempo que le dedican los padres: "Sarebbe lungo racontare quanto riguardo qui sia a noi padri necessario, quanta fatica per ciascu- 
por tanto, sin entrar en mayor debate, se acepta esta figura como una realidad cotidiana. Sin embargo, en estas obras en las que se defiende a ultranza la lactancia materna se ataca directamente este uso, se califica esa leche como "mercenaria" y se relatan las consecuencias de su uso con los tintes más negros: recurrir al ama de cría significa omisión de la maternidad $\mathrm{y}$, en algunos casos, se podría hablar casi de pecado, a no ser que se trate de madres enfermas ${ }^{33}$, con una cierta edad 0

no in tempo vi si duri prima che truovi quanto si conviene onesta, buona e faccente balia (...) sai quanto siano rare le buone nutrice e da molti richieste" (34). También Lionardo brevemente enumera las cualidades que tiene que tener un ama de cría: "io provederei, come tu di', d'avere balia buona, esperta e costumata, non per lasciar più ozio alla donna, non per torgli quella verso de' figliuoli devuta faccenda, ma per dare meno tristo nutrimento al fanciullo. E credo il vero che, oltre a quelle infermità, quali tu dicevi potevano dal corrotto latte venire" (37). En esta obra incluso Lionardo, que es el portavoz del autor y el que defiende sin cortapisas la lactancia materna, no puede dejar de señalar las cualidades que tiene que tener el ama de cría. Esto nos hace entender cómo realmente se trataba de una práctica social muy arraigada en la sociedad renacentista.

33. Alberti señala las pocas ocasiones en las que se puede recurrir a un ama de cría: "Piglierei questa licenza se la donna per sinistro alcuno fusse diventata debole" (1960: 37). Barbaro no se muestra en contra de esa costumbre, siempre que las madres lo hagan porque nos les quede otro remedio ("da giuste e honeste cagione impedite" (1548: 60r) y, a continuación, muestra cuáles deberían ser las cualidades de las amas de cría: "debbono almeno hauere grandissima auertenza, in far buona e diligente elettion della balia laquale deue essere di honesto lenaggio; non sciocca, non ebbriaca, non impudica: ma sauia, discreta, costumata, e da bene, affin che il tenero fanciullo non habbia da infettarsi di corrotti costumi, e a pigliare nel corpo e nell'animo certe maligne impressioni, lequali poi difficillimamente si possono estirpare" (60r). que no tengan leche, y por tanto, dar la leche a sus hijos suponga un peligro tanto para sus hijos como para ellas mismas (De Maio, 1988: 115).

En primer lugar, se alude al problema de la leche como vehículo de transmisión de las virtudes y cualidades morales, pero también de los vicios y enfermedades, de la mujer que amamanta. De esta manera, las amas de cría pasarían cualidades poco nobles a los bebés.

Como podemos concluir de estos textos, la leche es mucho más que la base de la alimentación del niño ya que influye sobre su salud, sí, pero también sobre su personalidad, su carácter, su educación, incluso su manera de hablar. En Breast Bottles and Babies, Fildes subraya que en esos tiempos se creía que la leche de la ama de cría "carried all her physical and mental qualities, her emotions, her food and drink, and her diseases" (1986: 203). Para los humanistas la leche mercenaria en general, pero sobre todo cuando el ama de cría no cumple con los requisitos mínimos, perjudica seriamente al noble, tanto en su infancia como en el resto de su vida. En este sentido declara Alberti: "Ancora più la nutrice non onesta, non costumata, sarà sufficiente ne' costumi del fanciullo nuocere e inclinallo a' vizii ed empierli l'animo di furiosi e bestiali passioni come d'iracundia, timidità, spaventi e simili mali" (1960: 37).

\section{Lactancia materna y el nuevo orden político y social en el Renacimiento}

En este artículo se han analizado los argumentos que Speroni presenta en su Discorso del lattare i figliuoli dalle madri (1542) 
a favor de la lactancia materna que tiene como base la naturaleza y el bien del hijo y de la familia y los que presenta en contra del ama de cría. Contrastando las palabras de Speroni con las de otros humanistas Barbaro, Alberti, Erasmo, Vives, Guevara, Luján y Fray Luis de León-, hemos podido constatar que los argumentos son los mismos, así como las fuentes en las que se basan; de hecho, para apoyar esta tesis de comportamiento femenino todos ellos se fundamentan en estudios fisiológicos sobre la leche materna, de obstetricia, ginecología y pediatría, pero también en tratados sobre comportamiento de la mujer publicados tanto durante el Renacimiento como en la Antigüedad. Podemos observar en estos textos un diálogo continuo en el que los clásicos y los intelectuales humanistas intentan convencer a las mujeres de darle el pecho a sus hijos y apartarles de una costumbre que carece de una base natural.

Tanta insistencia en que las mujeres cumplan con su deber como madres y den el pecho a sus hijos se justifica por el hecho de que la defensa a ultranza de la maternidad se revela elemento esencial para la formación del concepto de familia en la sociedad moderna, en el que el matrimonio y la descendencia se ha convertido en la base que hay que proteger por encima de todo. En esta nueva estructura social, apoyada tanto por el Estado como por la Iglesia, la mujer tiene un papel importante y su implicación es más intensa: la madre es responsable del sustento y el cuidado de los niños durante los primeros años de su vida.

Como hemos visto, tanto Speroni como otros humanistas europeos escriben estos diálogos, tratados o discursos con la intención evidente de concienciar a las madres sobre la necesidad de amaman- tar personalmente a sus hijos ${ }^{34} \mathrm{y}$, además, con el convencimiento de que las que mandan a sus hijos con amas de cría lo hacen o por ignorancia o por maldad (Speroni, 1740: 481) y, por tanto, hay un margen para educarlas en ese sentido. Todos estos textos son fundamentalmente formativos: hay que enseñar a las mujeres cómo se tienen que comportar como madres (madres completas y no medias madres o madrastras). Es evidente que la cuestión de la lactancia materna reviste una importancia mucho mayor que la de la simple nutrición o el cariño hacia el niño y contribuye a forjar una nueva concepción de la institución familiar, una idea moderna del matrimonio y de la función de la mujer dentro de él.

Instar a las mujeres a que amamanten a sus hijos significa defender a la familia, sobre todo en las clases altas, de todo tipo de influencias que vienen de fuera y que se pueden considerar altamente perjudiciales. Además, se deposita esa responsabilidad en la figura materna, dotándola así de una función importante para garantizar la institución familiar protegiéndola y controlándola desde su interior. Es verdad que, por un lado, se está dignificando el papel de la mujer, pero al mismo tiempo este se circunscribe de tal manera que

34. El público al que estaban dirigidos es evidente y explícito en los textos, las mujeres y, en concreto, en las obras de Speroni o de Erasmo las mujeres que acaban de dar a luz. Al final de Puerperio la joven madre Sofía se declara convencida de los beneficios, tanto para ella, como para el niño y la familia, de amamantar a su hijo, pero también es muy consciente de que esa decisión no le compete solo a ella; por eso, pide a su amigo que convenza a los demás (a su marido, a su familia), ya que ella no tiene la última palabra en este sentido: "A mí persuadido me has a hazer lo que te parece; querrá que lo mesmo persuadieses a mis padres y a mi marido" (Erasmo, 2005: 45). 
ese privilegio no suponga un perjuicio para los padres: estos van a mantener sus roles intactos a pesar de este claro enaltecimiento de la madre en la sociedad. Por tanto, no asistimos a un cambio en los roles y las funciones asignados a hombres y mujeres: estos se mantendrán prácticamente inalterados en el Renacimiento.

De hecho, la defensa de la lactancia materna tiene también otro tipo de repercusiones para la mujer: exigirle que se ocupase personalmente de amamantar a sus hijos la obligaba a permanecer dentro de los muros de la casa, la limitaba, todavía más, a su esfera privada, la alejaba del mundanal ruido (de las fiestas, de las reuniones y actividades sociales), pero también la dejaba al margen del ámbito laboral y cultura| ${ }^{35}$. En estos años se ve la necesidad de preservar la familia de todo peligro, mantener a la mujer estrictamente controlada en casa cumpliendo con sus obligaciones como madre y esposa, es decir, concentrada exclusivamente en sus funciones como reproductora y cuidadora de sus hijos y defendiendo a ultranza el honor familiar que depende de ella. La defensa de la lactancia tiene que ver directamente con este ámbito que es político, pero también económico, ya que la familia noble se considera una entidad política en el siglo XVI y tiene que funcionar de manera reglada porque tiene importantes repercusiones económicas. Es en este nuevo orden político, social y económico del Renacimiento en el que la defensa a ultranza de la lactancia mater-

35. En este sentido, Louis Hass (1998: 91) sostiene que la crítica de los humanistas a la delegación de la crianza de los hijos en una ama de cría se deba también a que esa práctica dejaba a las madres más libertad; esa libertad de la mujer se consideraba nociva para la familia. na por parte de tantos intelectuales encuentra su sentido pleno.

\section{Bibliografía}

Alberti, L.B. (1960). "I libri della famiglia, Libro primo", en C. Grayson (ed.) Opere volgari, Bari, Laterza, 13-82.

Alberti, L.B. (1971). The Albertis of Florence: L. B. Alberti's Della Famiglia, Lewisburg, Bucknell University Press.

Ariès, Ph. (1962). Centuries of Childhood: A Social History of Family Life, New York, Knopf.

Barbaro, F. (1548). Prvdentissimi et gravi docvmenti circa la elettion della moglie. Trad. M. Alberto Lollio Ferrarese. Venecia, Gabriel Giolito de Ferrari.

Basile, A. (2016). "Nota sull'uso di 'dimidiatus' (Gell., Noct. XII 1.6), Emerita, revista de Lingüística y Filología Clásica, LXXXIV, 1, 171-176.

Bergmann, E. (1996). "Monstrous Maternity in Fray Antonio de Guevara's Relox de Principes", en E.H. Friedman y C. (eds.) Larson Brave New Words: Studies in Spanish Golden Age Literature, New Orleans, UP of the South, 39-49.

Bruni, F. (1967). "Sperone Speroni e I'Accademia degli Infiammati", Filologia e Letteratura, a.XIII, n.49, vol.I, 24-71.

Cammarosano, F. (1920). La vita e le opere di Sperone Speroni, Empoli, Tipografia R. Noccioli.

Correll, B. (1990). "Malleable Material, Models of Power: Woman in Erasmus 'Marriageable Group' and Civility in Boys", English Literary History, vol. 57, 241-62.

De Maio, R. (1988). Mujer y Renacimiento, Madrid, Mondadori. 
Duby, G. y Perrot, M. (1992). Historia de las Mujeres. Volumen 3. Del Renacimiento a la Edad Moderna, Madrid, Taurus.

Erasmo de Rotterdam. (2005). "Puerperio”, en A. Herrán y M. Santos (eds.) Coloquios familiares. Erasmo de Rotterdam. Edición de Alonso Ruiz de Virués. BarceIona, Anthropos, 15-46.

Fano, A. (1909). Sperone Speroni (15001588): saggio sulla vita e sulle opere, $\mathrm{Pa}$ dua, Fratelli Drucker.

Fernández Valencia, A. (2011). "Cuerpo nutricio: iconografías de los discursos de lactación", en A. Fernández y M. López (eds.), Contar con el cuerpo. Construcciones de la identidad femenina, Madrid, Fundamentos, 167-205.

Fildes, V. (1986). Breast, Bottles, and Babies: A History of Infant Feeding, Oxford, Edinburgh UP.

Fildes, V. (1988). Wet-nursing: A History from Antiquity to the Present, Oxford, Basil Blackwell.

Fray Luis de León, (2006). La perfecta casada, Biblioteca virtual universal, Espasa Calpe, Madrid, https://biblioteca.org.ar/ libros/131489.pdf

Freccero, C. (2019). "Economy, Woman, and Renaissance Discourse", en M. Migiel y J. Schiesari (eds.) Refiguring Woman: Perspectives on Gender and the Italian Renaissance, Ithaca, NY, Cornell University Press, 192-208.

Guarino, G. A. (1971). "Introduction", en The Albertis of Florence: L. B. Alberti's Della Famiglia, Lewisburg, Bucknell University Press, pp. 7-26.

Guevara, Antonio de. (1994). Relox de príncipes. Ed. Emilio Blanco. Madrid, ABL editor.
Hairston, J. L. (2013). "The Economics of Milk and Blood in Alberti's Libri della famiglia. Maternal versus Wet-Nursing", en J.G. Sperling (ed.) Medieval and Renaissance Lactations. Images, Rhetorics, Practices, Hampshire College, USA, Ashgate, 187-212.

Hass, L. (1998). The Renaissance Man and His Children: Childbirth and Early Childhood in Florence, 1300-1600, Nueva York, Palgrave Macmillan.

Herlihy, D. y Klapisch-Zuber, Ch. (1985). Tuscans and Their Families. A Study of the Florentine Catasto of 1427, New Haven, CT, Yale UP.

Jordan, C. (1990). Renaissance Feminism: Literary Texts and Political Models, Ithaca, NY, Cornell University Press.

Kelly, J. (1999). Did Women Have A Renaissance?, en L. Hutson (ed.) Feminism and Renaissance Studies, Oxford, University of Oxford, 21-47.

King, M.L. (1993). Mujeres renacentistas. La búsqueda de un espacio, Madrid, Alianza.

Klapisch-Zuber, Ch. (1985). "Blood Parents and Milk Parents: Wet Nursing in Florence, 1300-1530", en Ch. KlapischZuber (ed.) Women, Family, and Ritual in Renaissance Italy, Chicago y Londres, The University of Chicago Press, 132-164.

Klapisch-Zuber, Ch. (1988). La famiglia e le donne nel Rinascimento a Firenze, Roma, Editori Laterza.

Kohl, G. y Witt, R.G. (1978). "On Wifely Duties", en B.G. Kohl. y G. Witt (eds.) The Earthly Republic: Italian Humanists on Government and Society, Philadelphia, University of Pennsylvania Press, 179230. 
Luján, P. de. (2010). Coloquios matrimoniales. Edición de Asunción e Asunción Rallo Gruss. Junta de Andalucía, Consejería de Cultura.

Matthews Grieco, S.F. (1991). "Breastfeeding, Wet Nursing and Infant Mortality in Europe (1400-1800)", en S.F. Matthews Grieco y C.A. Corsini Historical Perspectives on Breastfeeding, Florencia, UNICEF, $15-62$.

McLaren, D. (1985). "Marital fertility and lactation 1570-1720", en M. Prior (ed.) Women in English society 1500-1800, Londres, Routledge, 22-53.

Meneghin, A. (2010). "Nursing Infants and Wet-Nurses in Fifteenth-Century Florence: Piero Puro di Francesco da Vicchio and his Wife, Santa di Betto da San Benedetto", Fifteenth Century, vol. 9, 179-195.

Miller, N. y Yavneh, N. (2000). (eds.) Maternal Measures: Figuring Caregiving in the Early Modern Period, Burlington, VT, Ashgate.

Miller, N. y Yavneh, N. (2002). "Milking the Poor: Wet-nursing and the Sexual Economy of Early Modern Spain", en E. Lacarra (ed.) Sexuality, Love, and Marriage in Medieval and Early Renaissance Iberia, Nueva York, Routledge, 90-114.

Miles, M.R. (2008). The Secularization of the Breast (1350-1750), Londres, University of California.

Morant, I. (2002). Discursos de la vida buena. Matrimonio, mujer y sexualidad en la literatura humanista, Madrid, Cátedra.

Nadeau, C.A. (2001). "Blood Mother/Milk Mother: Breast-feeding, the Family, and the State in Antonio de Guevara's Relox de príncipes (Dial of Princes)", Hispanic Review, 69.2, 153-174.
Rivera, O. (2010). "Funciones de la madre en la ideología del género articulada en el coloquio 'Puerperio'”, Symposium, 64:1, 18-32.

Rivera, O. (2016). "La madre frente a la nodriza: Propiedades atribuidas a la leche materna en las obras humanistas", L'érudit franco-espagnol, volume 10, fall, 13-29.

Sánchez, T. (1996). La mujer sin identidad. Un ciclo vital de sumisión femenina durante el Renacimiento, Salamanca, Amarú.

Sandri, L. (1991). "Baliatico mercenario e abbandono dei bambini alle istituzioni assistenziali: un medesimo disagio sociale?", en M. G. Muzzarelli, P. Galetti y B. Andreolli (eds.) Donne e lavoro nell'ttalia medievale, Turín, Rosemberg \& Sellier, 93-104.

Scrivano, R. (1959). "Cultura e letteratura in Sperone Speroni", La Rassegna della Letteratura italiana, s. VIII, LXIII, 38-51.

Sperling, J.G. (ed.) (2013). Medieval and Renaissance Lactations: Images, Rhetorics, Farnham, Ashgate.

Speroni, S. (1740). "Discorso del lattare i figliuoli dalle madri", en Opere di M. Sperone Speroni degli Alvarotti tratte da' mss. Originali. Tomo secondo, Venecia, Domenico Occhi, 477-486.

Vianello, V. (1989). "Sperone Speroni: opere, stile e tradizione. Un ventennio di studi (1968-1988)", Quaderni Veneti, n.9, 203-222.

Vives, J.L. (1940). Instrucción de la mujer cristiana, Buenos Aires, Espasa-Calpe Argentina.

Zambetti, A. (1920). Della vita e delle opere di Sperone Speroni, Lecco, Arti Grafiche Lecchesi. 